\title{
Neonatal growth hormone deficiency
}

In contrast to children, neonates have high serum growth hormone (GH) levels during the first week of life. If normative data were available, this phenomenon might enable diagnosis of GH deficiency (GHD) without performing an invasive stimulation test. However, normative data have been lacking, as drawing blood from a healthy neonate for research purposes is deemed unethical.

Gerhard Binder (University Children's Hospital, Tuebingen, Germany) and colleagues used a highly sensitive enzymelinked immunosorbent assay to detect GH concentrations as low as $0.0016 \mathrm{ng} / \mathrm{ml}$, which allowed them to measure the GH content of the buffer eluate taken from blood dried on newborn screening cards-an almost 1:100 dilution of the original GH concentration. These cards were left over from standard screening procedures for metabolic disorders, performed 3-5 days after birth.

Binder et al. were thus able to provide $\mathrm{GH}$ reference values and determine a cut-off value for the diagnosis of GHD in neonates-a single measurement of a GH level <7 $\mu \mathrm{g} / \mathrm{l}$. Also, "dried GH was immunologically stable over time enabling reliable $\mathrm{GH}$ measurements in stored newborn screening cards and other filter papers after months or years," says Binder.

The investigators also observed a distinct sex dichotomy: healthy female neonates had significantly higher $\mathrm{GH}$ levels than healthy male neonates. This finding was known for rats, but highly disputed in humans and no data had so far been reported for children.

"Now, in analogy to congenital primary hypothyroidism and TSH measurement, GHD in the newborn can be established by a single $\mathrm{GH}$ measurement using the newborn screening card or by a blood analysis," concludes Binder.

Linda Koch

Original article Binder, G. et al. Rational approach to the diagnosis of severe growth hormone deficiency in the newborn. J. Clin. Endocrinol. Metab. 95, 2219-2226 (2010) 